\title{
Hysteroscopic images of early-stage endometrial tuberculosis
}

\author{
Dabao Xu $\cdot$ Min Xue $\cdot$ Xueying Han
}

Received: 12 August 2008 / Accepted: 11 September 2008/Published online: 7 October 2008

(C) Springer-Verlag 2008

\begin{abstract}
Endometrial tuberculosis (TB) is a disease that may cause uterine adhesion, amenorrhea, and subsequently infertility. Early diagnosis and treatment of endometrial TB is vital to better prognosis. We show in this paper a typical hysteroscopic image of early-stage endometrial TB. We hope hysteroscopists may benefit from the images detecting the early-stage of endometrial TB.
\end{abstract}

Keywords Hysteroscopy · Endometrial tuberculosis · Image

A 30-year-old woman, gravid 0 , para 0 , complained of failure to get pregnant since her marriage 8 years ago. Her menstruation, including the cycle, amount, and period, was normal. Her blood erythrocyte sedimentation rate was $40 \mathrm{~mm} / \mathrm{h}$; the hysterosalpingography showed that both of the oviducts were totally obstructed. Obsolete pulmonary tuberculosis was found on doing a chest radiograph. A Bultrasound scan showed an enhanced endometrial echo, and her husband's semen analysis was normal.

Hysteroscopy was carried out as a routine preparation before in vitro fertilization pre-embryo transfer, which revealed a normal cervical canal, uterine cavity, and a thin, smooth endometrium without apparent uterine adhesion. Both the uterine cornua and fallopian tubal ostia were visible. However, the radial enlarged vessels could be seen in the endometrium of the whole right uterine cornu area with a deformed right fallopian tubal ostium, and there was a yellow, superficial ulcer, about $3 \times 4 \mathrm{~mm}$ in size in the endometrium within the right cornua (Fig. 1). The left uterine cornu and left tubal ostium were normal from a hysteroscopic view. A hysteroscopic-targeted biopsy was taken from the ulcerated area, and the diagnosis of endometrial tuberculosis (TB) was confirmed pathologically.

D. Xu $\cdot$ M. Xue $(\bowtie) \cdot X$. Han

Department of Obstetrics and Gynecology,

the Third Xiangya Hospital, Central South University,

138 Tongzipo Road,

Changsha, Hunan 410013, China

e-mail: forxudabao@yahoo.com 


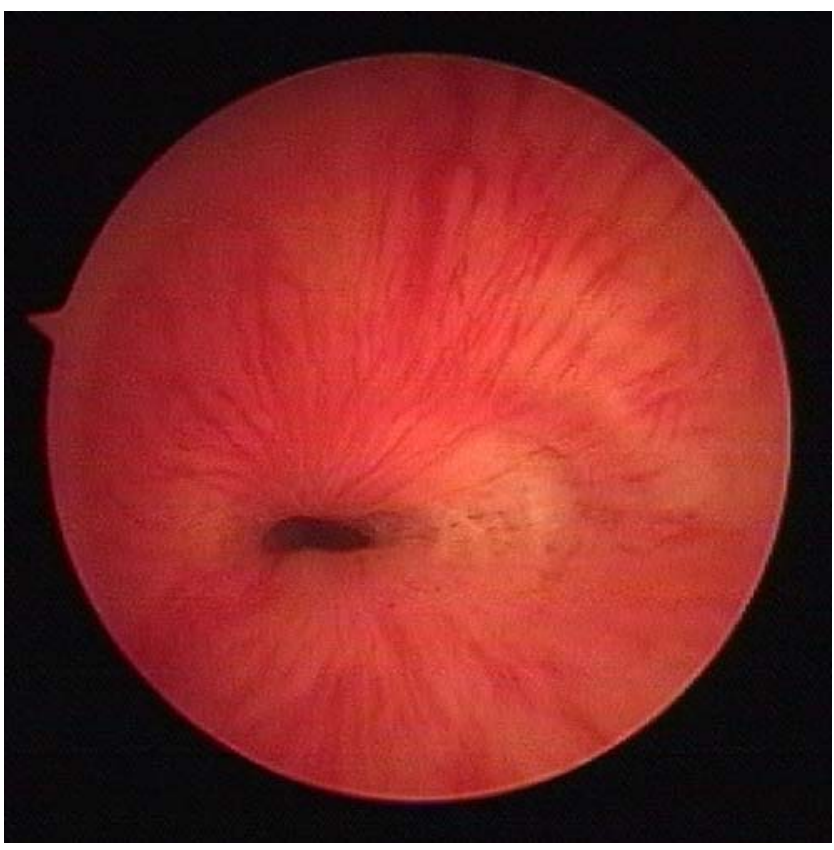

Fig. 1 Hysteroscopic image of early-stage endometial TB before antituberculosis treatment

A second-look hysteroscopy was performed after 9 months of anti-TB therapy and revealed that the once radial and enlarged vessels in the right cornu had reduced in size; however, the vessels still showed in

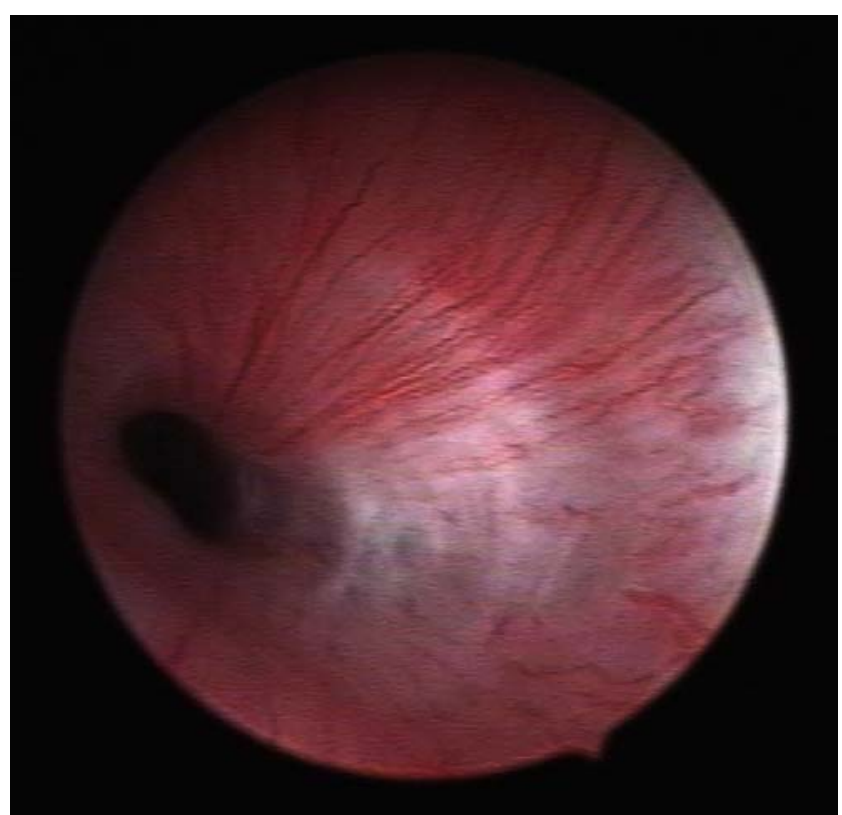

Fig. 2 Hysteroscopic image of early stage endometrial TB after antituberculosis treatment

radiation and there was no obvious ulcer (Fig. 2).The postoperative pathology indicated no evidence of active endometrial TB in the cornu, and the patient's menstruation was normal. 